\title{
Is cognitive training able to improve brain functioning with age?
}

\author{
Michael A Motes*,1 \\ ${ }^{1}$ School of Behavioral \& Brain Sciences, University of Texas at Dallas, 1966 Inwood Rd, Dallas, TX 75235, USA \\ *Author for correspondence: Tel.: +1 214905 3178; michael.motes@utdallas.edu
}

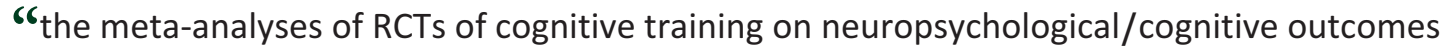
should be used to guide theoretical development and training/testing design optimization for

future RCT fMRI studies, and emphasis should be placed on associating neuropsychological/cognitive assessments of training-related change with fMRI-related change"
\end{abstract}

First draft submitted: 8 March 2018; Accepted for publication: 20 March 2018; Published online: 29 May 2018

Keywords: aging • cognitive strategy training $\bullet$ cognitive training $\bullet \mathrm{fMRl} \bullet$ functional magnetic resonance imaging $\bullet$ neuroimaging $\bullet$ senescence $\bullet$ training transfer $\bullet$ working memory training

Meta-analyses of randomized controlled trials (RCT) of cognitive training protocols have shown general and some specific cognitive and functional benefits of cognitive training in healthy older adults and adults with mild cognitive impairment (MCI) [1-5]. RCT functional neuroimaging (fMRI) studies also have revealed candidate neural systems underlying some of the cognitive training benefits [6-11]. However, although promising, considerable research is still needed to better understand optimal training domains and parameters, particularly, that facilitate transfer to daily functioning and to better understand the brain mechanisms supporting cognitive training benefits.

\section{Meta-analyses of RCTs of cognitive training in the older adults}

A meta-analysis of RCTs of various cognitive training protocols in older adults (RCTs $=31$ ) showed training-related benefits for overall cognitive function (Hedges' $\mathrm{g}=0.42)$, executive function $(\mathrm{g}=0.42)$, memory $(\mathrm{g}=0.35)$, attention $(\mathrm{g}=0.22)$ and visuospatial ability $(\mathrm{g}=0.18)$ [1]. Additionally, benefits were stronger for attention when training occurred over 8 weeks or more $(g=0.29$ vs $g=0.09$ ), and benefits were stronger for executive function when training occurred three times or more per week ( $g=0.65$ vs $g=0.26$ ) and there were 24 or more sessions total $(\mathrm{g}=0.70$ vs $\mathrm{g}=0.29$ ). However, no detectable benefits were found for individual versus group training or session durations greater than versus less than $60 \mathrm{~min}$. Another meta-analysis examining RCTs of commercially available computerized cognitive training programs in older adults (RCTs $=18$ ) showed cognitive training benefits for attention (Cohen's $\mathrm{d}=0.65$; although greater against passive, $\mathrm{d}=0.98$, than active, $\mathrm{d}=0.36$, control groups), visuospatial memory $(\mathrm{d}=0.25$, greater against passive, $\mathrm{d}=0.43$, than active, $\mathrm{d}=0.22$, controls), processing speed $(\mathrm{d}=0.29)$ and self-reported everyday function $(\mathrm{d}=0.28)$ [4]. However, no detectable benefits were found for executive function, memory, reasoning, verbal fluency or objectively measured everyday functions. Another metaanalysis specifically examining $n$-back working memory training (RCTs $=41$ ) showed cognitive training benefits for near transfer to working memory on $n$-back $(g=0.62)$ and other working memory tasks $(g=0.24)$ and far transfer to cognitive control $(\mathrm{g}=0.16)$ and fluid intelligence/visuospatial reasoning $(\mathrm{g}=0.16)$ [3]. On far transfer tasks, benefits were stronger against passive versus active controls, but benefits were not found to be moderated by the number of sessions, hours training, age or verbal versus nonverbal $n$-back training. Finally, a meta-analysis of RCTs of computerized cognitive training in older adults with MCI or dementia (MCI RCTs = 17; dementia RCTs = 12) revealed training-related benefits in MCI but not dementia [2]. In MCI, training-related benefits were observed for global cognition $(\mathrm{g}=0.38)$, verbal learning $(\mathrm{g}=0.39)$, verbal memory $(\mathrm{g}=0.42)$, nonverbal learning $(\mathrm{g}=0.50)$, working memory $(g=0.74)$, attention $(g=0.44)$ and psychosocial functioning $(g=0.52)$ [2]. No detectable benefits were found for nonverbal memory, executive function, processing speed, visuospatial skills, language, or daily living, and the observed differences were not found to be moderated by the use of active versus passive controls. General

Future $\because \cdots$ Medicine 
cognitive training benefits were also reported in a meta-analysis of pooled data on healthy older adults and MCI patients, with stronger benefits for working memory training over other training domains and for near over far transfer tasks [5]. In dementia, a weaker training-related benefit on overall cognition $(g=0.26)$ was observed, but the benefit was not significant $(\mathrm{g}=0.17)$ after removing two outlier studies [2].

\section{fMRI RCTs of cognitive training in older adults}

In fMRI research, two RCTs have examined the effects of working memory training in older adults [6,7]. In one RCT in healthy older adults, progressive training to increase visuospatial and verbal working memory span, compared with low-capacity working memory repetitive practice, led to training benefits in working memory capacity over the training sessions and transferred to sustained attention and episodic memory but not to inhibitory control or nonverbal reasoning [6]. Although progressive working memory training benefits were not shown to increase working memory capacity on tasks completed in the scanner, progressive working memory training led to decreased activation, under higher working memory demand, in right dorsolateral prefrontal, right superior temporal and bilateral occipital (i.e., lingual gyrus) cortices. In the other RCT in healthy older adults, progressive training on an $n$-back working memory task, compared with passive control, led to training benefits in $n$-back capacity and transferred to memory updating in an untrained delayed-recognition working memory task [7]. Progressive $n$-back training led to decreased activation, under higher working memory demand, in both the $n$-back and delayed recognition working memory tasks within left dorsolateral prefrontal cortex and decreased activation was associated with increased working memory capacity gains. Thus, across these two studies, progressive working memory training led to decreased activation in dorsolateral prefrontal cortex under higher working memory demand.

In an RCT in healthy older adults, variable-priority divided attention and fixed-priority divided attention training, compared with focused attention training, revealed a divided attention training benefit of reduced divided attention performance costs and a variable-priority divided attention training benefit of reduced performance costs moderated by attention priority [8]. Variable-priority training led to increased activation in right dorsolateral prefrontal cortex in dual-task conditions, and reduced post-training dual-task performance cost was associated with greater activation. Focused attention training, in contrast, led to reduced single-task activation bilaterally within dorsolateral prefrontal cortices, with shorter single-task reaction time associated with less activation. Thus, divided attention training was associated with increased activation in dorsolateral prefrontal cortex under divided attention conditions.

Another RCT in healthy elderly adults examining multicomponent cognitive control training, compared with passive control, revealed transfer of training to untrained measures of cognitive control, processing speed and general cognitive functioning but not episodic or working memory span [9]. On a task completed in the scanner, training also led to increased inhibitory control and to increased interference-related activation in right supramarginal gyrus, right precuneus and left insula. Thus, transfer of cognitive control training to an untrained cognitive control task led to increased activation in a subset of regions previously identified as being involved in cognitive control.

In MCI patients, an RCT examining combined multidomain cognitive and physical exercise training, compared with passive control, showed training-related improvement in Alzheimer's disease symptoms and in visuo-spatial recall and phonemic fluency [10]. Although training did not transfer to performance on a visuospatial attention task completed in the scanner, the passive control group at follow-up showed increased activation within visual attention regions (i.e., left middle temporal, intraparietal and premotor cortices), but the trained group did not show significant activation change. The authors suggested that cognitive training might have mitigated declines in neural systems involved in visual attention.

In an RCT in healthy older adults, cognitive strategy training (i.e., training designed to facilitate comprehension and abstraction), compared with passive and active (i.e., aerobic exercise) controls, led to near transfer to abstraction ability [11]. Although training did not transfer to performance on a processing speed task completed in the scanner, transfer was observed in that the cognitive training group showed increased involvement of left prefrontal cortex following cognitive training (i.e., an increase in the correlation between BOLD signal-change and the reaction time index of processing speed on the task; whereas the control groups showed a decrease in the correlation). Thus, cognitive strategy training led to increased involvement of dorsolateral prefrontal cortex on a far transfer measure of processing speed.

Finally, in an RCT in healthy older adults, goal orientation and management training, compared with active control (i.e., brain health education), led to training-related increased activation, within a network comprised of left parahippocampus and right dorsolateral prefrontal cortex, temporo-parietal junction and precuneus, that was 
related to selectively attending to stimuli (i.e., goal-directed attending to one category of sequentially stimuli) [12]. Additionally, increased network activation following training predicted subsequent memory for the goal-directed stimuli. Thus, higher order cognitive training led to increased activation of a network of brain regions including dorsolateral prefrontal cortex on a transfer measure of selective-attention.

\section{Conclusion}

The meta-analytic reviews have shown the general efficacy of cognitive training in healthy elderly adults and those with MCI. Although transfer of training to untrained cognitive domains has been shown, no clear evidence favoring a particular mode of training on far transfer has been shown, and further research is needed on far transfer effects to objective measures of daily functioning and to long-term follow-ups. One RCT, however, has reported 5- and 10-year follow-up training-related benefits on self-reported and simulated daily living activities, showing small to medium training benefits $(\mathrm{g}=0.29$ and $\mathrm{g}=0.38$ at 5 - and 10 -years, respectively) [13,14], and reported 6-year follow-up training-related benefits on reductions in at-fault motor vehicle accidents $(g=0.50)[15,16]$.

RCTs involving task-related $\mathrm{fMRI}$ that have been conducted have shown cognitive training to affect involvement of dorsolateral prefrontal cortex in tasks both near and far from the training paradigms $[6-8,11,12]$. However, training effects have been mixed regarding training-related increases [8] or decreases [7] in dorsolateral prefrontal cortex recruitment and even in the general recruitment of dorsolateral prefrontal cortex [9,10]. Thus, more research is needed examine training and testing parameters accounting for these discrepancies. Additionally, the meta-analyses of RCTs of cognitive training on neuropsychological/cognitive outcomes should be used to guide theoretical development and training/testing design optimization for future RCT fMRI studies, and emphasis should be placed on associating neuropsychological/cognitive assessments of training-related change with fMRI-related change.

\section{Financial \& competing interests disclosure}

The author has no relevant affiliations or financial involvement with any organization or entity with a financial interest in or financial conflict with the subject matter or materials discussed in the manuscript. This includes employment, consultancies, honoraria, stock ownership or options, expert testimony, grants or patents received or pending, or royalties.

No writing assistance was utilized in the production of this manuscript.

\section{References}

1. Chiu H-L, Chu H, Tsai J-C et al. The effect of cognitive-based training for the healthy older people: a meta-analysis of randomized controlled trials. PLoS ONE 12(5), e0176742 (2017).

2. Hill NT, Mowszowski L, Naismith SL, Chadwick VL, Valensuela M, Lampit A. Computerized cognitive training in older adults with mild cognitive impairment or dementia: a systematic review and meta-analysis. Am. J. Psychiatry 174(4), 329-340 (2017).

3. Soveri A, Antfolk J, Karlsson L, Salo B, Laine M. Working memory training revisited: A multi-level meta-analysis of n-back training studies. Psychon. Bull. Rev. 24(4), 1077-1096 (2017).

4. Tetlow A, Edwards JD. Systematic literature review and meta-analysis of commercially available computerized cognitive training among older adults. J. Cog. Enhanc. 1(4), 559-575 (2017).

5. Mewborn CM, Lindbergh CA, Miller LS. Cognitive interventions for cognitively healthy, mildly impaired, and mixed samples of older adults: a systematic review and meta-analysis of randomized-controlled trials. Neuropsychol. Rev. 27(4), 403-439 (2017).

6. Brehmer Y, Rieckmann A, Bellander M, Westerberg H, Fischer H, Backman L. Neural correlates of training-related working-memory gains in old age. Neuroimage 58(4), 1110-1120 (2011).

7. Heinzel S, Lorenz RC, Pelz P et al. Neural correlates of training and transfer effects in working memory in older adults. Neuroimage 134, 236-249 (2016).

8. Belleville S, Mellah S, de Boysson C, Demonet J-F, Bier B. The pattern and loci of training-induced brain changes in healthy older adults are predicted by the nature of the intervention. PLOS ONE 9(8), e102710 (2014).

9. Kim H, Chey J, Lee S. Effects of multicomponent training of cognitive control function and brain activation in older adults. Neurosci. Res. 124, 8-15 (2017).

10. Maffei L, Picano MG, Andreassi MG et al. Randomized trial on the effects of a combined physical/cognitive training in aged MCI subjects: The Train the Brain study. Sci. Rep. 7, 39471 (2017).

11. Motes MA, Yezhuvath US, Aslan S, Spence JS, Rypma B, Chapman SB. Higher-order cognitive training effects on processing speed-related neural activity: a randomized trial. Neurobiol. Aging 62, 72-81 (2018).

12. Adnan A, Chen AJW, Novakovic-Agopian T, D'Esposito M, Turner GR. Brain changes following executive control training in older adults. Neurorehabil. Neural Repair 31(10-11), 910-922 (2017). 
13. Willis SL, Tennstedt SL, Marsiske M et al. Long-term effects of cognitive training on everyday functional outcomes in older adults. JAMA 296(23), 2805-2814 (2006).

14. Rebok GW, Ball K, Guey LT et al. Ten-year effects of the advanced cognitive training for independent and vital elderly cognitive training trial on cognition and everyday functioning in older adults. J. Am. Geriatr. Soc. 62(1), 16-24 (2014).

15. Ball K, Edwards JD, Ross LA, McGwin Jr G. Cognitive training decreases motor vehicle collision involvement of older drivers. J. Am. Geriatr. Soc. 58(11), 2107-2113 (2010).

16. Mowszowski L, Lampit A, Walton CC, Naismith SL. Strategy-based cognitive training for improving executive functions in older adults: a systematic review. Neuropsychol. Rev. 26(3), 252-270 (2016). 